\title{
Generic distance-squared mappings on plane curves
}

\author{
Shunsuke ICHIKI \\ (Received February 6, 2020) \\ (Revised June 8, 2020)
}

\begin{abstract}
A distance-squared function is one of the most significant functions in the application of singularity theory to differential geometry. Moreover, distance-squared mappings are naturally extended mappings of distance-squared functions, wherein each component is a distance-squared function. In this paper, compositions of a given plane curve and generic distance-squared mappings on the plane into the plane are investigated from the viewpoint of stability.
\end{abstract}

\section{Introduction}

Throughout this paper, let $\ell$ and $n$ stand for positive integers. In this paper, unless otherwise stated, all manifolds and mappings belong to class $C^{\infty}$ and all manifolds are without boundary. Let $q=\left(q_{1}, \ldots, q_{n}\right) \in \mathbb{R}^{n}$ be a given point. The mapping $d_{q}: \mathbb{R}^{n} \rightarrow \mathbb{R}$ defined by

$$
d_{q}(x)=\sum_{i=1}^{n}\left(x_{i}-q_{i}\right)^{2}
$$

is called a distance-squared function, where $x=\left(x_{1}, \ldots, x_{n}\right)$. In [5], the following notion is investigated.

Definition 1. Let $p_{1}, \ldots, p_{\ell}$ be $\ell$ given points in $\mathbb{R}^{n}$. Set $p=$ $\left(p_{1}, \ldots, p_{\ell}\right) \in\left(\mathbb{R}^{n}\right)^{\ell}$. The mapping $D_{p}: \mathbb{R}^{n} \rightarrow \mathbb{R}^{\ell}$ defined by

$$
D_{p}=\left(d_{p_{1}}, \ldots, d_{p_{\ell}}\right)
$$

is called a distance-squared mapping.

We have the following motivation for investigating distance-squared mappings. Height functions and distance-squared functions have been investigated in detail so far, and they are useful tools in the applications of singularity theory to differential geometry (see [1]). A mapping in which each

The author was supported by JSPS KAKENHI Grant Numbers JP16J06911 and JP19J00650.

2010 Mathematics Subject Classification. Primary 57R45; Secondary 57R35.

Key words and phrases. distance-squared mapping, stability, immersion with normal crossings, curvature. 
component is a height function is nothing but a projection. Projections as well as height functions or distance-squared functions have been investigated so far. For example, in [6] (resp., [2]), compositions of generic projections and embeddings (resp., stable mappings) are investigated from the viewpoint of stability (for the definition of stability, refer to [3]). On the other hand, a mapping in which each component is a distance-squared function is a distancesquared mapping. Therefore, it is natural to investigate distance-squared mappings as well as projections.

In this paper, compositions of a given plane curve and generic distancesquared mappings on the plane into the plane are investigated from the viewpoint of stability.

A mapping $f: \mathbb{R}^{n} \rightarrow \mathbb{R}^{\ell}$ is said to be $\mathscr{A}$-equivalent to a mapping $g: \mathbb{R}^{n} \rightarrow \mathbb{R}^{\ell}$ if there exist diffeomorphisms $\varphi: \mathbb{R}^{n} \rightarrow \mathbb{R}^{n}$ and $\psi: \mathbb{R}^{\ell} \rightarrow \mathbb{R}^{\ell}$ such that $\psi \circ f \circ \varphi^{-1}=g$. For given points $x=\left(x_{1}, \ldots, x_{n}\right), y=\left(y_{1}, \ldots, y_{n}\right) \in$ $\mathbb{R}^{n}$, set

$$
\overrightarrow{x y}=\left(y_{1}-x_{1}, \ldots, y_{n}-x_{n}\right) .
$$

Given $\ell$ points $p_{1}, \ldots, p_{\ell} \in \mathbb{R}^{n}(1 \leq \ell \leq n+1)$ are said to be in general position if $\ell=1$ or $\overrightarrow{p_{1} p_{2}}, \ldots, \overrightarrow{p_{1} p_{\ell}}(2 \leq \ell \leq n+1)$ are linearly independent.

In [5], a characterization of distance-squared mappings is given as follows:

Proposition 1 ([5]). (1) Let $\ell, n$ be integers such that $2 \leq \ell \leq n$, and let $p_{1}, \ldots, p_{\ell} \in \mathbb{R}^{n}$ be in general position. Then, $D_{p}: \mathbb{R}^{n} \rightarrow \mathbb{R}^{\ell}$ is $\mathscr{A}$-equivalent to the mapping defined by $\left(x_{1}, \ldots, x_{n}\right) \mapsto\left(x_{1}, \ldots, x_{\ell-1}\right.$, $\left.x_{\ell}^{2}+\cdots+x_{n}^{2}\right)$.

(2) Let $\ell, n$ be integers such that $1 \leq n<\ell$, and let $p_{1}, \ldots, p_{\ell} \in \mathbb{R}^{n}$ be $\ell$ points such that $p_{1}, \ldots, p_{n+1}$ are in general position. Then, $D_{p}: \mathbb{R}^{n} \rightarrow$ $\mathbb{R}^{\ell}$ is $\mathscr{A}$-equivalent to the inclusion $\left(x_{1}, \ldots, x_{n}\right) \mapsto\left(x_{1}, \ldots, x_{n}, 0, \ldots, 0\right)$.

In the following, by $N$, we denote a manifold of dimension 1. A mapping $f: N \rightarrow \mathbb{R}^{2}$ is called a mapping with normal crossings if the mapping $f$ satisfies the following conditions.

(1) For any $y \in \mathbb{R}^{2},\left|f^{-1}(y)\right| \leq 2$, where $|A|$ is the number of elements of the set $A$.

(2) For any two distinct points $q_{1}, q_{2} \in N$ satisfying $f\left(q_{1}\right)=f\left(q_{2}\right)$, we have $\operatorname{dim}\left(d f_{q_{1}}\left(T_{q_{1}} N\right)+d f_{q_{2}}\left(T_{q_{2}} N\right)\right)=2$.

From Corollary 8 in [4], we have the following.

Proposition 2 ([4]). Let $\gamma: N \rightarrow \mathbb{R}^{2}$ be an injective immersion, where $N$ is a manifold of dimension 1 . Then, the set

$$
\left\{p \in \mathbb{R}^{2} \times \mathbb{R}^{2} \mid D_{p} \circ \gamma: N \rightarrow \mathbb{R}^{2} \text { is an immersion with normal crossings }\right\}
$$

is dense in $\mathbb{R}^{2} \times \mathbb{R}^{2}$. 
On the other hand, the purpose of this paper is to investigate whether the set

$$
\left\{p \in \gamma(N) \times \gamma(N) \mid D_{p} \circ \gamma: N \rightarrow \mathbb{R}^{2} \text { is an immersion with normal crossings }\right\}
$$

is dense in $\gamma(N) \times \gamma(N)$ or not. Here, note that $O$ is an open set of $\gamma(N) \times \gamma(N)$ if there exists an open set $O^{\prime}$ of $\mathbb{R}^{2} \times \mathbb{R}^{2}$ satisfying $O=O^{\prime} \cap$ $(\gamma(N) \times \gamma(N))$.

Let $\gamma: N \rightarrow \mathbb{R}^{2}$ be an immersion. We say that $\kappa: U \rightarrow \mathbb{R}$ is called the curvature of $\gamma$ on a coordinate neighborhood $(U, t)$ of $N$ if

$$
\kappa(t)=\frac{\operatorname{det}\left(\begin{array}{ll}
\frac{d \gamma_{1}}{d t}(t) & \frac{d^{2} \gamma_{1}}{d t^{2}}(t) \\
\frac{d \gamma_{2}}{d t}(t) & \frac{d^{2} \gamma_{2}}{d t^{2}}(t)
\end{array}\right)}{\left(\left(\frac{d \gamma_{1}}{d t}(t)\right)^{2}+\left(\frac{d \gamma_{2}}{d t}(t)\right)^{2}\right)^{3 / 2}},
$$

where $\gamma=\left(\gamma_{1}, \gamma_{2}\right)$. Note that for a given point $q \in N$, whether $\kappa(q)=0$ or not does not depend on the choice of a coordinate neighborhood.

Definition 2. Let $N$ be a manifold of dimension 1. We say that an immersion $\gamma: N \rightarrow \mathbb{R}^{2}$ satisfies $(*)$ if for any non-empty open set $U$ of $N$, there exists a point $q \in U$ satisfying $\kappa(q) \neq 0$, where $\kappa$ is the curvature of $\gamma$ on a coordinate neighborhood around $q$.

The main result in this paper is the following.

THEOREM 1. Let $\gamma: N \rightarrow \mathbb{R}^{2}$ be an injective immersion satisfying (*), where $N$ is a manifold of dimension 1 . Then, the set

$$
\left\{p \in \gamma(N) \times \gamma(N) \mid D_{p} \circ \gamma: N \rightarrow \mathbb{R}^{2} \text { is an immersion with normal crossings }\right\}
$$

is dense in $\gamma(N) \times \gamma(N)$.

If we drop the hypothesis $(*)$ in Theorem 1 , then the conclusion of Theorem 1 does not necessarily hold (see Examples 1 and 2 in Section 2).

In Theorem 1, if the mapping $D_{p} \circ \gamma: N \rightarrow \mathbb{R}^{2}$ is proper, then the immersion with normal crossings $D_{p} \circ \gamma: N \rightarrow \mathbb{R}^{2}$ is necessarily stable (see [3], p. 86). Thus, from Theorem 1, we get the following.

Corollary 1. Let $N$ be a compact manifold of dimension 1. Let $\gamma: N \rightarrow$ $\mathbb{R}^{2}$ be an embedding satisfying $(*)$. Then, the set

$$
\left\{p \in \gamma(N) \times \gamma(N) \mid D_{p} \circ \gamma: N \rightarrow \mathbb{R}^{2} \text { is stable }\right\}
$$

is dense in $\gamma(N) \times \gamma(N)$. 
In Section 2, Examples 1 and 2 are given. In Section 3, preliminaries for the proof of Theorem 1 are given. Section 4 is devoted to the proof of Theorem 1.

\section{Dropping the hypothesis $(*)$ in Theorem 1}

In this section, we will give two examples such that Theorem 1 without the hypothesis $(*)$ does not hold (see Examples 1 and 2).

Firstly, we prepare the following proposition, which is used in Example 1.

Proposition 3. Let $\gamma: N \rightarrow \mathbb{R}^{2}$ be a mapping, where $N$ is a manifold of dimension 1. Let $p_{1}, p_{2}$ be two points of $\mathbb{R}^{2}$. Then, a point $q \in N$ is a singular point of the mapping $D_{p} \circ \gamma: N \rightarrow \mathbb{R}^{2}\left(p=\left(p_{1}, p_{2}\right)\right)$ if and only if

$$
\overrightarrow{p_{1} \gamma(q)} \cdot \frac{d \gamma}{d t}(q)=0 \quad \text { and } \quad \overrightarrow{p_{2} \gamma(q)} \cdot \frac{d \gamma}{d t}(q)=0
$$

where $t$ is a local coordinate around the point $q$ and "." stands for the inner product in $\mathbb{R}^{2}$, that is, $p_{1}$ and $p_{2}$ are on the line normal to the curve $\gamma(N)$ at $\gamma(q)$.

Proof. Let $q$ be a point of $N$. The composition of $\gamma: N \rightarrow \mathbb{R}^{2}$ and $D_{p}: \mathbb{R}^{2} \rightarrow \mathbb{R}^{2}$ is given as follows:

$$
D_{p} \circ \gamma(q)=\left(\left(\gamma_{1}(q)-p_{11}\right)^{2}+\left(\gamma_{2}(q)-p_{12}\right)^{2},\left(\gamma_{1}(q)-p_{21}\right)^{2}+\left(\gamma_{2}(q)-p_{22}\right)^{2}\right),
$$

where $p_{1}=\left(p_{11}, p_{12}\right), p_{2}=\left(p_{21}, p_{22}\right)$ and $\gamma=\left(\gamma_{1}, \gamma_{2}\right)$.

Then, we have

$$
\begin{aligned}
\frac{d D_{p} \circ \gamma}{d t}(q)=2\left(\left(\gamma_{1}(q)-p_{11}\right) \frac{d \gamma_{1}}{d t}(q)+\left(\gamma_{2}(q)-p_{12}\right) \frac{d \gamma_{2}}{d t}(q),\right. & \left.\left(\gamma_{1}(q)-p_{21}\right) \frac{d \gamma_{1}}{d t}(q)+\left(\gamma_{2}(q)-p_{22}\right) \frac{d \gamma_{2}}{d t}(q)\right) \\
= & 2\left(\overrightarrow{p_{1} \gamma(q)} \cdot \frac{d \gamma}{d t}(q), \overrightarrow{p_{2} \gamma(q)} \cdot \frac{d \gamma}{d t}(q)\right),
\end{aligned}
$$

where $t$ is a local coordinate around the point $q$. Hence, a point $q$ is a singular point of the mapping $D_{p} \circ \gamma$ if and only if

$$
\left(\overrightarrow{p_{1} \gamma(q)} \cdot \frac{d \gamma}{d t}(q), \overrightarrow{p_{2} \gamma(q)} \cdot \frac{d \gamma}{d t}(q)\right)=(0,0) .
$$

Example 1. In this example, we use Proposition 3. Let $\gamma: S^{1} \rightarrow \mathbb{R}^{2}$ be an embedding such that $\gamma\left(S^{1}\right)$ is given by Figure 1. Here, note that there 


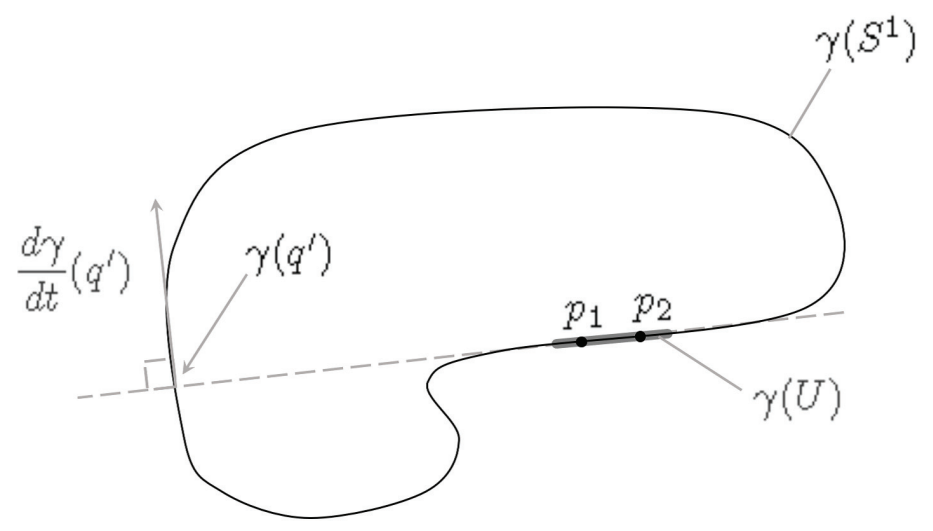

Fig. 1. Curve $\gamma$ of Example 1

exists an open set $U$ of $N$ such that for any $q \in U, \kappa(q)=0$ (see $\gamma(U)$ in Figure 1). Namely, $\gamma$ does not satisfy $(*)$.

Let $p=\left(p_{1}, p_{2}\right) \in \gamma(U) \times \gamma(U)$ be any point. Then, we will show that the mapping $D_{p} \circ \gamma$ is not an immersion. From Figure 1, it is clearly seen that

$$
\overrightarrow{p_{1} \gamma\left(q^{\prime}\right)} \cdot \frac{d \gamma}{d t}\left(q^{\prime}\right)=0 \quad \text { and } \quad \overrightarrow{p_{2} \gamma\left(q^{\prime}\right)} \cdot \frac{d \gamma}{d t}\left(q^{\prime}\right)=0
$$

where $\gamma\left(q^{\prime}\right)$ is the point in Figure 1 and $t$ is a local coordinate around the point $q^{\prime}$. By Proposition 3, the point $q^{\prime}$ is a singular point of $D_{p} \circ \gamma$. Namely, for any $p=\left(p_{1}, p_{2}\right) \in \gamma(U) \times \gamma(U)$, the mapping $D_{p} \circ \gamma$ is not an immersion. Since $\gamma(U) \times \gamma(U)$ is a non-empty open set of $\gamma\left(S^{1}\right) \times \gamma\left(S^{1}\right)$, the conclusion of Theorem 1 does not hold.

ExAmple 2. Let $I_{1}, I_{2}$ and $I_{3}$ be open intervals $(0,1),(1,2)$ and $(2,3)$ of $\mathbb{R}$, respectively. Let $\gamma: I_{1} \cup I_{2} \cup I_{3} \rightarrow \mathbb{R}^{2}$ be the mapping given by

$$
\gamma(t)= \begin{cases}(t,-1), & t \in I_{1}, \\ (t-1,0), & t \in I_{2}, \\ (t-2,1), & t \in I_{3} .\end{cases}
$$

For the image of $\gamma$, see Figure 2. Here, note that $\gamma$ does not satisfy $(*)$. Let $p=\left(p_{1}, p_{2}\right) \in \gamma\left(I_{2}\right) \times \gamma\left(I_{2}\right)$ be any point. Then, we will show that $D_{p} \circ \gamma$ is not a mapping with normal crossings. Since $p_{1}=\left(p_{11}, p_{12}\right), p_{2}=\left(p_{21}, p_{22}\right) \in \gamma\left(I_{2}\right)$, we have $p_{12}=p_{22}=0$. Thus, we obtain

$$
D_{p}\left(x_{1}, x_{2}\right)=\left(\left(x_{1}-p_{11}\right)^{2}+x_{2}^{2},\left(x_{1}-p_{21}\right)^{2}+x_{2}^{2}\right) .
$$




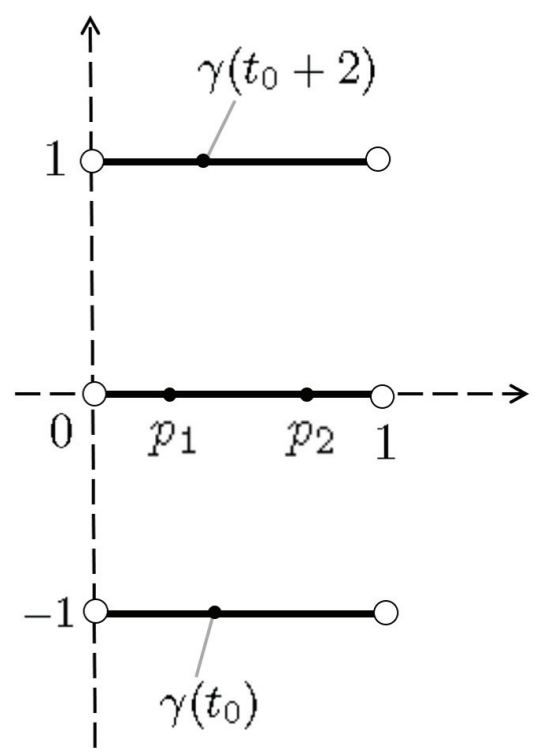

Fig. 2. Image of the mapping $\gamma$ of Example 2

Let $t_{0} \in I_{1}$ be any element. Then, it follows that $t_{0}+2 \in I_{3}$ and

$$
\left(D_{p} \circ \gamma\right)\left(t_{0}\right)=\left(D_{p} \circ \gamma\right)\left(t_{0}+2\right) \text {. }
$$

Since

$$
\begin{aligned}
& \left.\left(D_{p} \circ \gamma\right)\right|_{I_{1}}(t)=\left(\left(t-p_{11}\right)^{2}+1,\left(t-p_{21}\right)^{2}+1\right), \\
& \left.\left(D_{p} \circ \gamma\right)\right|_{I_{3}}(t)=\left(\left(t-2-p_{11}\right)^{2}+1,\left(t-2-p_{21}\right)^{2}+1\right),
\end{aligned}
$$

we get

$$
\begin{gathered}
d\left(D_{p} \circ \gamma\right)_{t_{0}}=2\left(\begin{array}{l}
t-p_{11} \\
t-p_{21}
\end{array}\right)_{t=t_{0}}, \\
d\left(D_{p} \circ \gamma\right)_{t_{0}+2}=2\left(\begin{array}{l}
t-2-p_{11} \\
t-2-p_{21}
\end{array}\right)_{t=t_{0}+2} .
\end{gathered}
$$

Since the rank of the $2 \times 2$ matrix $\left(d\left(D_{p} \circ \gamma\right)_{t_{0}}, d\left(D_{p} \circ \gamma\right)_{t_{0}+2}\right)$ is less than two, $D_{p} \circ \gamma$ is not a mapping with normal crossings. Hence, for any $p=\left(p_{1}, p_{2}\right) \in$ $\gamma\left(I_{2}\right) \times \gamma\left(I_{2}\right), D_{p} \circ \gamma$ is not a mapping with normal crossings.

REMARK 1. There is an example such that Theorem 1 without the hypothesis $(*)$ holds. Let $\gamma: \mathbb{R} \rightarrow \mathbb{R}^{2}$ be the mapping defined by $\gamma(t)=(t, 0)$. Set 


$$
\begin{aligned}
A=\left\{p \in \gamma(\mathbb{R}) \times \gamma(\mathbb{R}) \mid D_{p} \circ \gamma: \mathbb{R} \rightarrow \mathbb{R}^{2}\right. \text { is an immersion } \\
\\
\text { with normal crossings }\} .
\end{aligned}
$$

We will show that $A$ is dense in $\gamma(\mathbb{R}) \times \gamma(\mathbb{R})$. Let $p_{1}=\left(p_{11}, p_{12}\right), p_{2}=$ $\left(p_{21}, p_{22}\right) \in \gamma(\mathbb{R})(=\mathbb{R} \times\{0\})$ be arbitrary points. Then, we have

$$
D_{p} \circ \gamma(t)=\left(\left(t-p_{11}\right)^{2},\left(t-p_{21}\right)^{2}\right),
$$

where $p=\left(p_{1}, p_{2}\right)$. It is not hard to see that if $p_{11} \neq p_{21}$, then there exists a diffeomorphism $H: \mathbb{R}^{2} \rightarrow \mathbb{R}^{2}$ such that $H \circ D_{p} \circ \gamma(t)=(t, 0)$. Namely, if $p_{11} \neq p_{21}$, then $D_{p} \circ \gamma$ is an immersion with normal crossings. On the other hand, if $p_{11}=p_{21}$, then $D_{p} \circ \gamma$ is not an immersion with normal crossings. Hence,

$$
A=\left\{p \in \gamma(\mathbb{R}) \times \gamma(\mathbb{R}) \mid p_{11} \neq p_{21}\right\} .
$$

Thus, $A$ is dense in $\gamma(\mathbb{R}) \times \gamma(\mathbb{R})$.

\section{Preliminaries for the proof of Theorem 1}

For the proof of Theorem 1, we prepare Proposition 4 and Lemma 1.

Proposition 4. Let $L$ be a straight line of $\mathbb{R}^{2}$. For any $p_{1}, p_{2} \in L$ $\left(p_{1} \neq p_{2}\right)$ and for any $\tilde{p}_{1}, \tilde{p}_{2} \in L\left(\tilde{p}_{1} \neq \tilde{p}_{2}\right)$, there exists an affine transformation $H: \mathbb{R}^{2} \rightarrow \mathbb{R}^{2}$ such that

$$
H \circ D_{p}=D_{\tilde{p}}
$$

where $p=\left(p_{1}, p_{2}\right)$ and $\tilde{p}=\left(\tilde{p}_{1}, \tilde{p}_{2}\right)$.

ProOF. Set $p_{1}=\left(p_{11}, p_{12}\right), \quad p_{2}=\left(p_{21}, p_{22}\right), \quad \tilde{p}_{1}=\left(\tilde{p}_{11}, \tilde{p}_{12}\right) \quad$ and $\quad \tilde{p}_{2}=$ $\left(\tilde{p}_{21}, \tilde{p}_{22}\right)$.

Let $H_{1}: \mathbb{R}^{2} \rightarrow \mathbb{R}^{2}$ be the linear transformation defined by

$$
H_{1}\left(X_{1}, X_{2}\right)=\left(X_{1}, X_{1}-X_{2}\right) \text {. }
$$

Then, we have

$$
\begin{aligned}
H_{1} \circ D_{p}\left(x_{1}, x_{2}\right)= & \left(\left(x_{1}-p_{11}\right)^{2}+\left(x_{2}-p_{12}\right)^{2},\right. \\
& \left.2\left(\left(p_{21}-p_{11}\right) x_{1}+\left(p_{22}-p_{12}\right) x_{2}\right)+c_{1}\right),
\end{aligned}
$$

where $c_{1}$ is a constant term.

Let $H_{2}: \mathbb{R}^{2} \rightarrow \mathbb{R}^{2}$ be the affine transformation defined by

$$
H_{2}\left(X_{1}, X_{2}\right)=\left(X_{1}, X_{2}-c_{1}\right) \text {. }
$$


Then, we get

$$
\begin{aligned}
H_{2} \circ H_{1} \circ D_{p}\left(x_{1}, x_{2}\right)= & \left(\left(x_{1}-p_{11}\right)^{2}+\left(x_{2}-p_{12}\right)^{2},\right. \\
& \left.2\left(\left(p_{21}-p_{11}\right) x_{1}+\left(p_{22}-p_{12}\right) x_{2}\right)\right) .
\end{aligned}
$$

Since $p_{1}, p_{2}, \tilde{p}_{1}, \tilde{p}_{2} \in L$ and $p_{1} \neq p_{2}$, there exist $\lambda_{1}, \lambda_{2} \in \mathbb{R}$ satisfying

$$
\begin{aligned}
& \tilde{p}_{1}=p_{1}+\lambda_{1} \overrightarrow{p_{1} p_{2}}, \\
& \tilde{p}_{2}=p_{1}+\lambda_{2} \overrightarrow{p_{1} p_{2}} .
\end{aligned}
$$

Since $\tilde{p}_{1} \neq \tilde{p}_{2}$, we get $\lambda_{1} \neq \lambda_{2}$.

Let $H_{3}: \mathbb{R}^{2} \rightarrow \mathbb{R}^{2}$ be the linear transformation defined by

$$
H_{3}\left(X_{1}, X_{2}\right)=\left(X_{1}-\lambda_{1} X_{2}, X_{1}-\lambda_{2} X_{2}\right) \text {. }
$$

Then, we get

$$
\begin{aligned}
H_{3} \circ H_{2} \circ H_{1} \circ D_{p}\left(x_{1}, x_{2}\right) & \\
= & \left(x_{1}^{2}-2\left(p_{11}+\lambda_{1}\left(p_{21}-p_{11}\right)\right) x_{1}+x_{2}^{2}-2\left(p_{12}+\lambda_{1}\left(p_{22}-p_{12}\right)\right) x_{2}+d_{1},\right. \\
& \left.x_{1}^{2}-2\left(p_{11}+\lambda_{2}\left(p_{21}-p_{11}\right)\right) x_{1}+x_{2}^{2}-2\left(p_{12}+\lambda_{2}\left(p_{22}-p_{12}\right)\right) x_{2}+d_{2}\right),
\end{aligned}
$$

where $d_{1}, d_{2}$ are constant terms. By (1) and (2), we also get

$$
\begin{aligned}
H_{3} \circ & H_{2} \circ H_{1} \circ D_{p}\left(x_{1}, x_{2}\right) \\
& =\left(x_{1}^{2}-2 \tilde{p}_{11} x_{1}+x_{2}^{2}-2 \tilde{p}_{12} x_{2}+d_{1}, x_{1}^{2}-2 \tilde{p}_{21} x_{1}+x_{2}^{2}-2 \tilde{p}_{22} x_{2}+d_{2}\right) \\
& =\left(\left(x_{1}-\tilde{p}_{11}\right)^{2}+\left(x_{2}-\tilde{p}_{12}\right)^{2}+d_{1}^{\prime},\left(x_{1}-\tilde{p}_{21}\right)^{2}+\left(x_{2}-\tilde{p}_{22}\right)^{2}+d_{2}^{\prime}\right),
\end{aligned}
$$

where $d_{1}^{\prime}, d_{2}^{\prime}$ are constant terms.

Let $H_{4}: \mathbb{R}^{2} \rightarrow \mathbb{R}^{2}$ be the affine transformation defined by

$$
H_{4}\left(X_{1}, X_{2}\right)=\left(X_{1}-d_{1}^{\prime}, X_{2}-d_{2}^{\prime}\right) \text {. }
$$

Then, we have

$$
\begin{aligned}
H_{4} \circ & H_{3} \circ H_{2} \circ H_{1} \circ D_{p}\left(x_{1}, x_{2}\right) \\
& =\left(\left(x_{1}-\tilde{p}_{11}\right)^{2}+\left(x_{2}-\tilde{p}_{12}\right)^{2},\left(x_{1}-\tilde{p}_{21}\right)^{2}+\left(x_{2}-\tilde{p}_{22}\right)^{2}\right) \\
& =D_{\tilde{p}}\left(x_{1}, x_{2}\right) .
\end{aligned}
$$

This completes the proof of Proposition 4.

Lemma 1. Let $\gamma: N \rightarrow \mathbb{R}^{2}$ be an immersion satisfying $(*)$, where $N$ is a manifold of dimension 1. Then, for any non-empty open set $U_{1} \times U_{2}$ of $N \times N$, 
there exists an element $\left(q_{1}, q_{2}\right) \in U_{1} \times U_{2}$ such that

$$
\operatorname{det}\left(\begin{array}{ll}
\frac{d \gamma_{1}}{d t_{1}}\left(q_{1}\right) & \gamma_{1}\left(q_{2}\right)-\gamma_{1}\left(q_{1}\right) \\
\frac{d \gamma_{2}}{d t_{1}}\left(q_{1}\right) & \gamma_{2}\left(q_{2}\right)-\gamma_{2}\left(q_{1}\right)
\end{array}\right) \neq 0
$$

where $\gamma=\left(\gamma_{1}, \gamma_{2}\right)$ and $t_{1}$ is a local coordinate around $q_{1}$.

Proof. Let $U_{1} \times U_{2}$ be any non-empty open set of $N \times N$. Then, there exists a coordinate neighborhood $\left(U_{1}^{\prime} \times U_{2}^{\prime},\left(t_{1}, t_{2}\right)\right)$ satisfying $U_{1}^{\prime} \times U_{2}^{\prime} \subset$ $U_{1} \times U_{2}$. Fix $q_{1}^{\prime} \in U_{1}^{\prime}$.

Now, suppose that for any point $t_{2} \in U_{2}^{\prime}$,

$$
\operatorname{det}\left(\begin{array}{ll}
\frac{d \gamma_{1}}{d t_{1}}\left(q_{1}^{\prime}\right) & \gamma_{1}\left(t_{2}\right)-\gamma_{1}\left(q_{1}^{\prime}\right) \\
\frac{d \gamma_{2}}{d t_{1}}\left(q_{1}^{\prime}\right) & \gamma_{2}\left(t_{2}\right)-\gamma_{2}\left(q_{1}^{\prime}\right)
\end{array}\right)=0
$$

where $\gamma=\left(\gamma_{1}, \gamma_{2}\right)$. By (3), we have

$$
\frac{d \gamma_{1}}{d t_{1}}\left(q_{1}^{\prime}\right)\left(\gamma_{2}\left(t_{2}\right)-\gamma_{2}\left(q_{1}^{\prime}\right)\right)-\frac{d \gamma_{2}}{d t_{1}}\left(q_{1}^{\prime}\right)\left(\gamma_{1}\left(t_{2}\right)-\gamma_{1}\left(q_{1}^{\prime}\right)\right)=0,
$$

for any point $t_{2} \in U_{2}^{\prime}$. Hence, we get

$$
\begin{gathered}
\frac{d \gamma_{1}}{d t_{1}}\left(q_{1}^{\prime}\right) \frac{d \gamma_{2}}{d t_{2}}\left(t_{2}\right)-\frac{d \gamma_{2}}{d t_{1}}\left(q_{1}^{\prime}\right) \frac{d \gamma_{1}}{d t_{2}}\left(t_{2}\right)=0, \\
\frac{d \gamma_{1}}{d t_{1}}\left(q_{1}^{\prime}\right) \frac{d^{2} \gamma_{2}}{d t_{2}^{2}}\left(t_{2}\right)-\frac{d \gamma_{2}}{d t_{1}}\left(q_{1}^{\prime}\right) \frac{d^{2} \gamma_{1}}{d t_{2}^{2}}\left(t_{2}\right)=0,
\end{gathered}
$$

for any point $t_{2} \in U_{2}^{\prime}$. By (4) and (5), we have

$$
\left(\begin{array}{cc}
\frac{d \gamma_{2}}{d t_{2}}\left(t_{2}\right) & -\frac{d \gamma_{1}}{d t_{2}}\left(t_{2}\right) \\
\frac{d^{2} \gamma_{2}}{d t_{2}^{2}}\left(t_{2}\right) & -\frac{d^{2} \gamma_{1}}{d t_{2}^{2}}\left(t_{2}\right)
\end{array}\right)\left(\begin{array}{l}
\frac{d \gamma_{1}}{d t_{1}}\left(q_{1}^{\prime}\right) \\
\frac{d \gamma_{2}}{d t_{1}}\left(q_{1}^{\prime}\right)
\end{array}\right)=\left(\begin{array}{l}
0 \\
0
\end{array}\right),
$$

for any point $t_{2} \in U_{2}^{\prime}$. Since $\gamma$ is an immersion, it follows that

$$
\left(\begin{array}{l}
\frac{d \gamma_{1}}{d t_{1}}\left(q_{1}^{\prime}\right) \\
\frac{d \gamma_{2}}{d t_{1}}\left(q_{1}^{\prime}\right)
\end{array}\right) \neq\left(\begin{array}{l}
0 \\
0
\end{array}\right)
$$


By (6) and (7), we have

$$
\operatorname{det}\left(\begin{array}{cc}
\frac{d \gamma_{2}}{d t_{2}}\left(t_{2}\right) & -\frac{d \gamma_{1}}{d t_{2}}\left(t_{2}\right) \\
\frac{d^{2} \gamma_{2}}{d t_{2}^{2}}\left(t_{2}\right) & -\frac{d^{2} \gamma_{1}}{d t_{2}^{2}}\left(t_{2}\right)
\end{array}\right)=0
$$

for any point $t_{2} \in U_{2}^{\prime}$. This contradicts the hypothesis that $\gamma$ satisfies $(*)$.

REMARK 2. It is clearly seen that Lemma 1 does not depend on the choice of a coordinate neighborhood containing a point $q_{1}$ of $N$.

\section{Proof of Theorem 1}

Let $O$ be any non-empty open set of $\gamma(N) \times \gamma(N)$. Then, there exist nonempty open sets $O_{1}$ and $O_{2}$ of $\gamma(N)$ satisfying $O_{1} \times O_{2} \subset O$. For the proof, it is sufficient to show that there exist points $p_{1} \in O_{1}$ and $p_{2} \in O_{2}$ such that $D_{p} \circ \gamma: N \rightarrow \mathbb{R}^{2}$ is an immersion with normal crossings, where $p=\left(p_{1}, p_{2}\right)$. Since $\gamma$ is continuous, there exist coordinate neighborhoods $\left(U_{1}, t_{1}\right)$ and $\left(U_{2}, t_{2}\right)$ of $N$ such that $\gamma\left(U_{1}\right) \subset O_{1}$ and $\gamma\left(U_{2}\right) \subset O_{2}$.

Now, let $I_{1}$ (resp., $I_{2}$ ) be an open interval containing 0 (resp., 1) of $\mathbb{R}$, and let $\Phi: U_{1} \times U_{2} \times I_{1} \times I_{2} \rightarrow \mathbb{R}^{4}$ be the mapping defined by

$$
\begin{aligned}
\Phi\left(t_{1}, t_{2}, s_{1}, s_{2}\right)= & \left(\gamma\left(t_{1}\right)+s_{1} \overrightarrow{\gamma\left(t_{1}\right) \gamma\left(t_{2}\right)}, \gamma\left(t_{1}\right)+s_{2} \overrightarrow{\gamma\left(t_{1}\right) \gamma\left(t_{2}\right)}\right) \\
= & \left(\left(1-s_{1}\right) \gamma_{1}\left(t_{1}\right)+s_{1} \gamma_{1}\left(t_{2}\right),\left(1-s_{1}\right) \gamma_{2}\left(t_{1}\right)+s_{1} \gamma_{2}\left(t_{2}\right),\right. \\
& \left.\left(1-s_{2}\right) \gamma_{1}\left(t_{1}\right)+s_{2} \gamma_{1}\left(t_{2}\right),\left(1-s_{2}\right) \gamma_{2}\left(t_{1}\right)+s_{2} \gamma_{2}\left(t_{2}\right)\right),
\end{aligned}
$$

where $\gamma=\left(\gamma_{1}, \gamma_{2}\right)$. Then, we get

$$
J \Phi_{\left(t_{1}, t_{2}, s_{1}, s_{2}\right)}=\left(\begin{array}{cccc}
\left(1-s_{1}\right) \frac{d \gamma_{1}}{d t_{1}}\left(t_{1}\right) & s_{1} \frac{d \gamma_{1}}{d t_{2}}\left(t_{2}\right) & \gamma_{1}\left(t_{2}\right)-\gamma_{1}\left(t_{1}\right) & 0 \\
\left(1-s_{1}\right) \frac{d \gamma_{2}}{d t_{1}}\left(t_{1}\right) & s_{1} \frac{d \gamma_{2}}{d t_{2}}\left(t_{2}\right) & \gamma_{2}\left(t_{2}\right)-\gamma_{2}\left(t_{1}\right) & 0 \\
\left(1-s_{2}\right) \frac{d \gamma_{1}}{d t_{1}}\left(t_{1}\right) & s_{2} \frac{d \gamma_{1}}{d t_{2}}\left(t_{2}\right) & 0 & \gamma_{1}\left(t_{2}\right)-\gamma_{1}\left(t_{1}\right) \\
\left(1-s_{2}\right) \frac{d \gamma_{2}}{d t_{1}}\left(t_{1}\right) & s_{2} \frac{d \gamma_{2}}{d t_{2}}\left(t_{2}\right) & 0 & \gamma_{2}\left(t_{2}\right)-\gamma_{2}\left(t_{1}\right)
\end{array}\right) .
$$

Set $s_{1}=0$ and $s_{2}=1$. Then, we have 


$$
J \Phi_{\left(t_{1}, t_{2}, 0,1\right)}=\left(\begin{array}{cccc}
\frac{d \gamma_{1}}{d t_{1}}\left(t_{1}\right) & 0 & \gamma_{1}\left(t_{2}\right)-\gamma_{1}\left(t_{1}\right) & 0 \\
\frac{d \gamma_{2}}{d t_{1}}\left(t_{1}\right) & 0 & \gamma_{2}\left(t_{2}\right)-\gamma_{2}\left(t_{1}\right) & 0 \\
0 & \frac{d \gamma_{1}}{d t_{2}}\left(t_{2}\right) & 0 & \gamma_{1}\left(t_{2}\right)-\gamma_{1}\left(t_{1}\right) \\
0 & \frac{d \gamma_{2}}{d t_{2}}\left(t_{2}\right) & 0 & \gamma_{2}\left(t_{2}\right)-\gamma_{2}\left(t_{1}\right)
\end{array}\right) .
$$

Let us first show that there exists an element $\left(\tilde{t}_{1}, \tilde{t}_{2}\right) \in U_{1} \times U_{2}$ such that $\operatorname{det} d \Phi_{\left(\tilde{t}_{1}, \tilde{t}_{2}, 0,1\right)} \neq 0$. Let $\varphi_{1}: U_{1} \times U_{2} \rightarrow \mathbb{R}$ and $\varphi_{2}: U_{1} \times U_{2} \rightarrow \mathbb{R}$ be the functions defined by

$$
\begin{gathered}
\varphi_{1}\left(t_{1}, t_{2}\right)=\operatorname{det}\left(\begin{array}{ll}
\frac{d \gamma_{1}}{d t_{1}}\left(t_{1}\right) & \gamma_{1}\left(t_{2}\right)-\gamma_{1}\left(t_{1}\right) \\
\frac{d \gamma_{2}}{d t_{1}}\left(t_{1}\right) & \gamma_{2}\left(t_{2}\right)-\gamma_{2}\left(t_{1}\right)
\end{array}\right), \\
\varphi_{2}\left(t_{1}, t_{2}\right)=\operatorname{det}\left(\begin{array}{ll}
\frac{d \gamma_{1}}{d t_{2}}\left(t_{2}\right) & \gamma_{1}\left(t_{2}\right)-\gamma_{1}\left(t_{1}\right) \\
\frac{d \gamma_{2}}{d t_{2}}\left(t_{2}\right) & \gamma_{2}\left(t_{2}\right)-\gamma_{2}\left(t_{1}\right)
\end{array}\right),
\end{gathered}
$$

respectively. Note that the function $\varphi_{1}$ (resp., $\varphi_{2}$ ) is defined by the entries of the 1 st column vector and the 3 rd column vector of $J \Phi_{\left(t_{1}, t_{2}, 0,1\right)}$ (resp., the 2nd column vector and the 4 th column vector of $\left.J \Phi_{\left(t_{1}, t_{2}, 0,1\right)}\right)$. In order to show that there exists an element $\left(\tilde{t}_{1}, \tilde{t}_{2}\right) \in U_{1} \times U_{2}$ such that det $d \Phi_{\left(\tilde{t}_{1}, \tilde{t}_{2}, 0,1\right)} \neq 0$, it is sufficient to show that there exists an element $\left(\tilde{t}_{1}, \tilde{t}_{2}\right) \in U_{1} \times U_{2}$ satisfying $\varphi_{1}\left(\tilde{t}_{1}, \tilde{t}_{2}\right) \neq 0$ and $\varphi_{2}\left(\tilde{t}_{1}, \tilde{t}_{2}\right) \neq 0$. By Lemma 1 , there exists $\left(t_{1}^{\prime}, t_{2}^{\prime}\right) \in U_{1} \times U_{2}$ such that $\varphi_{1}\left(t_{1}^{\prime}, t_{2}^{\prime}\right) \neq 0$. Since the function $\varphi_{1}$ is continuous, there exists an open neighborhood $U_{1}^{\prime} \times U_{2}^{\prime}\left(\subset U_{1} \times U_{2}\right)$ of $\left(t_{1}^{\prime}, t_{2}^{\prime}\right)$ satisfying $\varphi_{1}\left(t_{1}, t_{2}\right) \neq 0$ for any $\left(t_{1}, t_{2}\right) \in U_{1}^{\prime} \times U_{2}^{\prime}$. Moreover, by Lemma 1 , there exists $\left(\tilde{t}_{1}, \tilde{t}_{2}\right) \in U_{1}^{\prime} \times U_{2}^{\prime}$ such that $\varphi_{2}\left(\tilde{t}_{1}, \tilde{t}_{2}\right) \neq 0$. Namely, there exists an element $\left(\tilde{t}_{1}, \tilde{t}_{2}\right) \in U_{1} \times U_{2}$ such that $\operatorname{det} d \Phi_{\left(\tilde{t}_{1}, \tilde{t}_{2}, 0,1\right)} \neq 0$.

Now, by the inverse function theorem, there exists an open neighborhood $V$ of $\left(\tilde{t}_{1}, \tilde{t}_{2}, 0,1\right) \in U_{1} \times U_{2} \times I_{1} \times I_{2}$ such that $\Phi: V \rightarrow \Phi(V)$ is a diffeomorphism. Let $\Sigma \subset \mathbb{R}^{2} \times \mathbb{R}^{2}$ be the set consisting of points $p=\left(p_{1}, p_{2}\right) \in \mathbb{R}^{4}$ such that $D_{p} \circ \gamma: N \rightarrow \mathbb{R}^{2}$ is not an immersion with normal crossings. Note that by Proposition 2 , the set $\mathbb{R}^{4}-\Sigma$ is dense in $\mathbb{R}^{4}$. Set

$$
\Delta=\left\{\left(y_{1}, y_{2}\right) \in \mathbb{R}^{2} \times \mathbb{R}^{2} \mid y_{1}=y_{2}\right\} .
$$

Since $\Phi(V)$ is an open set of $\mathbb{R}^{4}$ and the set $\Delta$ is a proper algebraic set of $\mathbb{R}^{4}$, there exists an element $p^{\prime}=\left(p_{1}^{\prime}, p_{2}^{\prime}\right) \in \Phi(V)-\Sigma \cup \Delta$. As $p^{\prime} \notin \Sigma$, the com- 
position $D_{p^{\prime}} \circ \gamma: N \rightarrow \mathbb{R}^{2}$ is an immersion with normal crossings. Set $\left(t_{1}^{\prime}, t_{2}^{\prime}\right.$, $\left.s_{1}^{\prime}, s_{2}^{\prime}\right)=\left(\left.\Phi\right|_{V}\right)^{-1}\left(p_{1}^{\prime}, p_{2}^{\prime}\right)$. Then, we have

$$
\begin{aligned}
& p_{1}^{\prime}=\gamma\left(t_{1}^{\prime}\right)+s_{1}^{\prime} \overrightarrow{\gamma\left(t_{1}^{\prime}\right) \gamma\left(t_{2}^{\prime}\right)}, \\
& p_{2}^{\prime}=\gamma\left(t_{1}^{\prime}\right)+s_{2}^{\prime} \overrightarrow{\gamma\left(t_{1}^{\prime}\right) \gamma\left(t_{2}^{\prime}\right)} .
\end{aligned}
$$

Since $p_{1}^{\prime} \neq p_{2}^{\prime}$, we get $\gamma\left(t_{1}^{\prime}\right) \neq \gamma\left(t_{2}^{\prime}\right)$. Let $L$ be the straight line defined by

$$
L=\left\{\gamma\left(t_{1}^{\prime}\right)+s \overrightarrow{\left(t_{1}^{\prime}\right) \gamma\left(t_{2}^{\prime}\right)} \mid s \in \mathbb{R}\right\} .
$$

Set $\tilde{p}_{1}=\gamma\left(t_{1}^{\prime}\right)$ and $\tilde{p}_{2}=\gamma\left(t_{2}^{\prime}\right)$. Then, it is clearly seen that $\tilde{p}_{1} \in O_{1}$ and $\tilde{p}_{2} \in O_{2}$. Since $p_{1}^{\prime}, p_{2}^{\prime} \in L\left(p_{1}^{\prime} \neq p_{2}^{\prime}\right)$ and $\tilde{p}_{1}, \tilde{p}_{2} \in L\left(\tilde{p}_{1} \neq \tilde{p}_{2}\right)$, by Proposition 4 , there exists an affine transformation $H: \mathbb{R}^{2} \rightarrow \mathbb{R}^{2}$ such that

$$
H \circ D_{p^{\prime}}=D_{\tilde{p}},
$$

where $\tilde{p}=\left(\tilde{p}_{1}, \tilde{p}_{2}\right)$. Since $D_{p^{\prime}} \circ \gamma: N \rightarrow \mathbb{R}^{2}$ is an immersion with normal crossings, $D_{\tilde{p}} \circ \gamma: N \rightarrow \mathbb{R}^{2}$ is also an immersion with normal crossings.

\section{Acknowledgement}

The author is most grateful to the anonymous reviewer for carefully reading the first manuscript of this paper and for giving invaluable suggestions. The author is grateful to Takashi Nishimura for his kind comments.

\section{References}

[1] J. W. Bruce and P. J. Giblin, Curves and singularities (second edition), Cambridge University Press, Cambridge, 1992.

[2 ] J. W. Bruce and N. P. Kirk, Generic projections of stable mappings, Bull. London Math. Soc., 32 (2000), 718-728.

[3] M. Golubitsky and V. Guillemin, Stable mappings and their singularities, Graduate Texts in Mathematics 14, Springer, New York, 1973.

[4] S. Ichiki, Composing generic linearly perturbed mappings and immersions/injections, J. Math. Soc. Japan, 70 (2018), no. 3, 1165-1184.

[5] S. Ichiki and T. Nishimura, Distance-squared mappings, Topology Appl., 160 (2013), 1005-1016.

[6] J. N. Mather, Generic projections, Ann. of Math., (2) 98 (1973), 226-245.

Shunsuke Ichiki

Department of Mathematical and Computing Science School of Computing, Tokyo Institute of Technology

Tokyo 152-8552, Japan

E-mail: ichiki@c.titech.ac.jp 